\title{
REPARTO DE RECURSOS DE LOS GREMIOS DE LAGARTIJAS EN EL CAÑÓN DE CHÍNIPAS, CHIHUAHUA, MÉXICO
}

\author{
Enrique SANTOYO-BRITO' ${ }^{1}$ y Julio A. LEMOS-ESPINAL ${ }^{2}$
}

1Colegio de Postgraduados. Postgrado en Recursos Genéticos y Productividad-Ganadería. Carretera México Texcoco, Km. 36.5 C.P. 56230, Montecillo, Mpio. de Texcoco, Edo. de México, MÉXICO: e.santoyobrito@gmail.com.

2Universidad Nacional Autónoma de México, Unidad de Biología, Tecnología y Prototipos. Facultad de Estudios Superiores Iztacala. Avenida de los Barrios \# 1, Los Reyes Iztacala. Tlalnepantla, Edo. de México, C. P. 54090, MÉXICO. lemos@servidor.unam.mx.

Santoyo-Brito, E. \& J. A. Lemos-Espinal. 2010. Reparto de recursos de los gremios de lagartijas en el Cañón de Chínipas, Chihuahua, México. Acta Zool. Mex. (n.s.) 26(2): 435-450.

RESUMEN. Se estudió el reparto de dos recursos -espacio y tiempo- entre los ensambles de lagartijas en tres asociaciones vegetales (bosque tropical caducifolio [BTC], bosque de encino [BE] y bosque de pino [BP]) en el Cañón de Chínipas, Chihuahua, en las que en conjunto se observaron dieciséis especies de diez géneros. El traslape de nicho se evaluó utilizando el índice Pianka y la amplitud de nicho al emplear la fórmula de Simpson. Los resultados indican que los valores del índice de traslape de nicho, por pares de especies, por lo general son menores a $O_{j k}=0.50$. Sin embargo, para las pseudocomunidades de lagartijas generadas con la información recabada en el BTC y la del BP sí se obtuvieron valores estadísticos que sugieren la presencia de la posible competencia respecto al recurso tiempo. Al evaluar los valores de amplitud de nicho indican que los individuos estudiados tienden a ser especialistas al utilizar el recurso tiempo y generalistas en cuanto al espacio. Sceloporus clarkii fue la única especie registrada en las tres asociaciones vegetales.

Palabras clave: Chihuahua, Ecología, Recursos, Reptiles, Lagartijas

Santoyo-Brito, E. \& J. A. Lemos-Espinal. 2010. Resource partitioning in lizards assemblages in the Chinipas Canyon, Chihuahua, Mexico. Acta Zool. Mex. (n.s.) 26(2): 435-450.

ABSTRACT. We studied the resource partitioning of time and space in lizard assemblages in three different plant associations (tropical deciduous forest [BTC], oak forest [BE], and pine forest [BP]) located in Chínipas' Canyon, Chihuahua, in which we observed sixteen lizard species of ten genera. The niche overlap was determined using Pianka's index while Simpson's formula was used for the calculation of the niche breadth. The results indicate that the niche overlap index values, per species pair, are generally lower than $O_{j k}=0.50$. However, for the pseudo communities, generated with the survey information gathered from topical deciduous forests and pine forests, statistical values were obtained that suggest the existence of potential competition in regards to resource time in each vegetation association. The niche breadth values indicate that the individuals

Recibido: 08/10/2009; aceptado: 13/04/2010. 
tend to be specialists when using time resource and generalists when using resource space. Sceloporus clarkii was the only species recorded in the three plant associations.

Key words: Chihuahua, Ecology, Resources, Reptiles, Lizards

\section{INTRODUCCIÓN}

Uno de los temas más estudiados en ecología ha sido el determinar cómo las especies difieren en el uso de los recursos (Luiselli 2008). Por lo general se establece entre miembros de grupos de especies simpátricas que cuentan con alguna semejanza en cuanto a sus papeles o roles tróficos (Schoener 1986). De manera tradicional para su estudio, el reparto de recursos, se divide en tres categorías dimensionales, el hábitat, el alimento y el tiempo (Schoener 1968; Pianka 1969, 1973, 1975), mismas que pueden subdividirse en macrohábitat, microhábitat, tipo y tamaño de alimento y tiempo de actividad estacional y diaria (Schoener 1974). En los estudios de las interacciones de especies y de la estructura de los ensambles es útil cuantificar el grado de traslape entre pares de especies que se presentan al considerar diversos recursos (Hurlbert 1978; Lawlor 1980) así como el grado de especialización. Es por esto que su principal propósito es analizar los límites que la competencia interespecífica impone en cuanto al número de especies que de manera estable pueden coexistir (Pianka 1974; Schoener 1974). Sin embargo, debe tomarse en cuenta que la medición y la interpretación del traslape de nicho entre especies consumidoras del mismo recurso depende de lo que se está comparando entre ellas; por ejemplo, la morfología, el uso de cierto microhábitat, el tiempo u horario de forrajeo, el comportamiento de alimentación y los recursos consumidos (Lawlor 1980). La mayoría de estos estudios únicamente documentan las diferencias pero no analizan los mecanismos que las causan, ya que de manera conjunta o por medio de un sólo estudio ambas metas son difíciles de alcanzar (Toft 1985). Por ejemplo, las medidas de traslape de nicho están diseñadas para medir el grado en que dos especies comparten sólo un recurso o el conjunto de recursos comunes (Lawlor 1980) y no expresan el grado de la posible competencia (Sale 1974). Evaluar la competencia requiere por lo menos una de dos aproximaciones: 1) la experimental, que demanda perturbar el sistema al añadir o remover individuos o 2) la observacional, que involucra el uso de modelos y trata de implicar la competencia de manera indirecta (Schoener 1974). Es por esto que comúnmente primero se documenta el fenómeno. Un problema con los estudios del reparto de recursos es que difieren en cuanto a causas y patrones entre grupos de diferentes organismos, por lo que se dificulta hacer generalizaciones. Sin embargo, para ciertos grupos de animales estos estudios son abundantes (Luiselli 2008), por ejemplo, en anfibios y reptiles (Toft 1985). Para el caso de estudios realizados en México que involucran el reparto de recursos, sólo por mencionar algunos, se encuentran Lemos-Espinal \& Franco-López (1984), Altamirano \& García (1989), Altamirano et al. (1990, 1992), Lara \& Castro-Franco 
(1991), Ortega et al. (1992, 1994, 1995), Álvarez y Sarabia (2006, 2007), García de la Peña et al. (2007) y Macip-Ríos \& Muñoz-Alonso (2008).

En el suroeste de Chihuahua se localiza el Cañón de Chínipas, que por el excelente estado de conservación en que se encuentran sus áreas ribereñas, selvas y los bosques de las laderas, la Comisión Nacional para el Conocimiento y uso de la Biodiversidad (CONABIO) designó como la Región Terrestre Prioritaria RTP-32. El Cañón funciona como corredor entre la biota de la Sierra Madre Occidental (SMO) hacia el norte y el sur, así como entre elementos de la sierra y la costa. Además, la orografía y topografía accidentada del área, las asociaciones vegetales, la baja densidad humana y el complicado acceso al sitio, son características peculiares del cañón (Lemos-Espinal \& Smith 2007).

Sobre los anfibios y reptiles del Cañón de Chínipas, prácticamente sólo se conoce el listado y no hay estudios ecológicos para la herpetofauna de esta zona de Chihuahua. Por lo anterior, el objetivo del presente trabajo fue determinar el traslape $\left(O_{j k}\right)$ de nicho entre los pares de especies de saurios registrados en el Cañón de Chínipas así como el grado de amplitud de nicho $\left(D_{s}\right)$ de las especies.

\section{Área de estudio}

\section{MATERIAL Y MÉTODOS}

El Cañón de Chínipas se localiza en la ecorregión de las Sierras Templadas y se ubica en la SMO en la subprovincia denominada Gran Meseta y Cañones Chihuahuenses. La RTP32 se conforma principalmente por tres municipios: Chínipas, Guazapares y Uruachi. Sus coordenadas extremas son: $27^{\circ} 02^{\prime} 24^{\prime \prime}$ a $27^{\circ} 46^{\prime} 48^{\prime \prime} \mathrm{N}$ y $108^{\circ} 09^{\prime} 00^{\prime \prime}$ a $108^{\circ} 40^{\prime} 48^{\prime \prime} \mathrm{O}$ (Arriaga et al. 2000) y la altitud en esta zona varía de los $200 \mathrm{~m}$ en la parte más profunda del Cañón de Chínipas (Barranca del Septentrión), hasta los 3,300 m en la cima del Cerro Mohinora. La extensión del Cañón es de 1459 km², y casi su totalidad, a excepción del Valle de Chínipas, se caracteriza por la presencia de barrancas profundas. El área de estudio muestra diferentes asociaciones vegetales. En la parte más baja de la región (alrededores del Valle de Chínipas y Barranca del Septentrión, de 200-1200 m de altitud), se encuentran bosques tropicales caducifolios (BTC) caracterizados por la presencia de Bursera fagaroides, Guazuma ulmifolia, Ipomoea arborescens y Lysiloma ivaricada, entre otras. A medida que se asciende, estos bosques se mezclan con cactáceas columnares para posteriormente cambiar a bosques de encino (BE) (Quercus chihuahuensis), hasta un punto en donde se observan masas puras de esta especie (1200-1750 m de altitud). En la partes altas (1750-2500 m de altitud) se presenta el bosque de pino (BP) conformado por varias especies (Pinus ayacahuite, P. durangensis, P. engelmanni y P. leiophylla).

\section{Muestreo de la herpetofauna}

De marzo a noviembre del 2007 se realizaron visitas mensuales -cada visita se consideró como una unidad muestral- de 12 días de duración a 30 puntos (25 
localidades, tres cauces de ríos y dos afluentes) distribuidos en los municipios del área de estudio. Las localidades se eligieron con base en dos mapas topográficos, realizados por INEGI en el 2005, que muestran las asociaciones vegetales. El primer mapa utilizado fue el de ciudad Obregón, escala 1:250,000, que abarca información de Sonora, Sinaloa, Chihuahua y el área de Chínipas. El segundo mapa fue el de Chínipas, escala 1:50,000. La altitud y las posibilidades de acceso, al tomar en cuenta el conocimiento de pobladores locales, fueron determinantes en la selección de las localidades.

En cada localidad para realizar los muestreos se establecieron cuatro trayectos aleatorios (de longitud variable -100 a 200 m- según las condiciones y estructura del hábitat de cada localidad para un total de 120 trayectos). Los trayectos fueron visitados por dos personas por lo menos dos veces al día. El horario de muestreo fue de 8:00 a 19:00 h y para registrar lagartijas del género Phyllodactylus en el BTC, se realizaron muestreos nocturnos de 19:00 a 1:00 h.

\section{Análisis de datos}

Traslape del nicho. Para determinar la división en el uso del espacio (microhábitats) y el horario de actividad diaria de las especies de lagartijas en las tres asociaciones de vegetación, se usó el método propuesto por Winemiller \& Pianka (1990), en el cual se construyen matrices que contienen datos de uso de los microhábitats y horario de actividad diaria en las columnas, mientras que las especies observadas se disponen en las filas.

Para examinar el traslape del nicho se empleó el algoritmo de aleatorización de ceros RA3 utilizando el programa EcoSim (Winemiller \& Pianka1990; Vitt \& Carvalho 1995; Vitt \& Zani 1996) el cual genera 1000 pseudocomunidades en las que se calculan nuevas superposiciones de nicho, la media del traslape observado, la media del traslape simulado y la probabilidad de que el traslape observado $\geq$ esperado $(\alpha=0.05)$ (Gotelli \& Entsminger 2001). El algoritmo empleado conserva la amplitud de nicho de cada especie, pero aleatoriza los recursos que usa. Se emplea cuando se desea mantener el grado de especialización de cada especie (Joern \& Lawlor 1980; Lawlor 1980; Gotelli \& Entsminger 2001).

El uso del microhábitat y horario de actividad de las especies se comparó usando la ecuación de traslape del nicho propuesta por Pianka (1973), con la que se obtiene un valor de 0 cuando no existe traslape de la dimensión evaluada entre los pares de especies valoradas y valor de 1 cuando el traslape es máximo (Gotelli \& Entsminger 2001). Su ecuación es la siguiente:

$$
O_{j k}=\frac{\left(\sum p_{i j} p_{i k}\right)}{\left(\sqrt{\sum p_{i j}{ }^{2} \sum{p_{i k}}^{2}}\right)}
$$


Donde:

$O_{j k}=$ traslape en una de las dimensiones del nicho entre las especie $j \mathrm{y}$ la especie $k$

$p_{i j}=$ valor de importancia del recurso $i$ para la especie $j$

$p_{i k}=$ valor de importancia del recurso $i$ para la especie $k$

Amplitud del nicho. La diferencia en el uso del microhábitat y en el horario de actividad de las especies de lagartijas en las tres asociaciones vegetales, se evaluó a través de la modificación de la ecuación de Simpson (1949) (citado en Witthaker \& Levin 1976):

$$
D_{s}=\frac{\left[\left(\sum p_{i}^{2}\right)^{-1}-1\right]}{(N-1)}
$$

Donde:

$D_{s}=$ Amplitud en una de las dimensiones del nicho

$p_{i}=$ Valor de importancia del recurso $i$ para la especie

$N=$ Número de recursos disponibles

Los valores de este índice tienden a 0 cuando los organismos son selectivos y a 1 cuando son generalistas (Álvarez \& Sarabia 2006, 2007).

\section{RESULTADOS}

Se enlistaron 10 géneros y 16 especies de lagartijas, sólo Sceloporus clarkii se registró en las tres asociaciones vegetales del Cañón de Chínipas (Cuadro 1). En las tres asociaciones vegetales se determinaron los microhábitats: "base de arbusto", "sobre suelo", "orilla de arroyo", "sobre roca", "bajo roca", "sobre tocón", "bajo tocón”, "sobre árbol” y "poste de cerca" (Cuadro 2).

Uso de espacio en el BTC. Se registraron siete géneros y diez especies; ninguna ocupó todos los microhábitats. Nueve de las diez especies se ubicaron en "sobre roca" y sólo una "bajo tocón" (Cuadro 2). Los valores del traslape estimado varían de 0 a 0.98 (Cuadro 3). Para las pseudocomunidades la media del traslape observado $=0.31$ y la media del traslape simulado $=0.25$. La probabilidad de que el traslape observado $\geq$ esperado $=0.089$. Los resultados de amplitud de nicho mostraron que los individuos tienden a ser especialistas, el valor más alto fue 0.29 (Cuadro 4).

Uso de espacio en el BE. En los microhábitats del BE se observaron seis géneros y siete especies. No se registró alguna especie ocupando todos los microhábitats Cuatro de las siete especies se registraron en "sobre roca" y en "base de arbusto", "orilla de río" y 
"bajo tocón" sólo se registró una especie (Cuadro 2). Los valores del traslape del uso de espacio en el BE indicaron que por lo general es bajo, sólo cinco valores son superiores a 0.05 , siendo 0.92 el valor más alto (Cuadro 5). Para las pseudocomunidades la media del traslape observado $=0.193$ y la media del traslape simulado $=0.203$. La probabilidad de que el traslape observado $\geq$ esperado $=0.494$. Los valores estimados de amplitud de nicho fueron bajos; el valor más alto fue 0.15 (Cuadro 4) lo que indica que los individuos de estas especies tienden a ser especialistas.

Uso de espacio en el BP. Se registraron seis géneros y siete especies, ninguna ocupó todos los microhábitat. Cinco de las siete especies ocuparon "bajo roca" y en "base de arbusto" y "sobre suelo" se registró el menor número de especies (Cuadro 2). En el BP se obtuvo el valor de sobreposición máxima entre Plestiodon callicephalus/P. parviauriculatus. En algunos pares de especies el traslape fue nulo (Cuadro 6). Para las pseudocomunidades la media del traslape observado $=0.288$ y la media del traslape simulado $=0.247$. La probabilidad de que el traslape observado $\geq$ esperado $=0.219$. Los valores de la amplitud de nicho indican la tendencia a ser especialistas, el valor máximo estimado fue de 0.27 (Cuadro 4).

Cuadro 1. Especies de saurios y número de registros, en tres asociaciones vegetales $(\mathrm{BTC}=$ bosque tropical, $\mathrm{BE}=$ bosque de encino y $\mathrm{BP}=$ bosque de pino) en el Cañón de Chínipas, Chihuahua.

\begin{tabular}{lcccc}
\hline & \multicolumn{3}{c}{ Asociación vegetal } & Total \\
Especie & BTC & BE & BP & \\
\hline Anolis nebulosus & 46 & 12 & - & 58 \\
Aspidoscelis costata & 291 & 224 & - & 551 \\
Ctenosaura macrolopha & 163 & - & - & 163 \\
Elgaria kingii & - & - & 15 & 15 \\
Heloderma horridum & 10 & 4 & - & 14 \\
Holbrookia elegans & 194 & - & - & 194 \\
Phyllodactylus tuberculosus & 21 & - & - & 21 \\
Plestiodon callicephalus & - & 2 & 2 & 4 \\
Plestiodon parviauriculatus & - & 1 & 2 & 3 \\
Sceloporus albiventris & 235 & - & - & 235 \\
Sceloporus clarkii & 119 & 56 & 51 & 226 \\
Sceloporus jarrovii & - & - & 209 & 209 \\
Sceloporus lemosespinali & - & - & 46 & 46 \\
Sceloporus nelsoni & 145 & - & - & 145 \\
Sceloporus virgatus & - & - & 16 & 16 \\
Urosaurus bicarinatus & 59 & 35 & - & 94 \\
Total & 1283 & 334 & 341 & 1994 \\
\hline
\end{tabular}


Cuadro 2. Número de registros de las especies de saurios observadas en los microhábitats en el bosque tropical caducifolio (BTC), bosque de encino (BE) y bosque de pino (BP) en el Cañón de Chínipas, Chihuahua.

\begin{tabular}{|c|c|c|c|c|c|c|c|c|c|}
\hline \multicolumn{10}{|c|}{ Microhábitats } \\
\hline Especies & $\begin{array}{l}\text { Base de } \\
\text { arbusto }\end{array}$ & $\begin{array}{c}\text { Sobres } \\
\text { suelo }\end{array}$ & $\begin{array}{c}\text { Orilla } \\
\text { de arroyo }\end{array}$ & $\begin{array}{c}\text { Sobre } \\
\text { roca }\end{array}$ & $\begin{array}{l}\text { Bajo } \\
\text { roca }\end{array}$ & $\begin{array}{l}\text { Sobre } \\
\text { tocón }\end{array}$ & $\begin{array}{l}\text { Bajo } \\
\text { tocón }\end{array}$ & $\begin{array}{l}\text { Sobre } \\
\text { árbol }\end{array}$ & $\begin{array}{c}\text { Poste } \\
\text { de cerca }\end{array}$ \\
\hline \multicolumn{10}{|l|}{ BTC } \\
\hline P. tuberculosus & - & - & - & - & 21 & & - & - & - \\
\hline H. horridum & 1 & 6 & - & 1 & - & 1 & - & 1 & - \\
\hline A. nebulosus & - & - & - & 3 & - & 3 & - & 22 & 18 \\
\hline C. macrolopha & - & - & 3 & 16 & - & 27 & - & 71 & 46 \\
\hline H. elegans & 76 & 56 & 43 & 19 & - & & - & - & - \\
\hline S. albiventris & - & - & 14 & 5 & - & 59 & - & 110 & 47 \\
\hline S. clarkii & - & - & - & 14 & 2 & 15 & 3 & 73 & 12 \\
\hline S. nelsoni & - & 5 & 7 & 131 & - & 2 & - & - & - \\
\hline U. bicarinatus & - & - & - & 5 & - & 12 & - & 39 & 3 \\
\hline A. costata & 79 & 146 & 48 & 5 & - & 13 & - & - & - \\
\hline \multicolumn{10}{|l|}{$\mathrm{BE}$} \\
\hline H. horridum & - & 3 & - & 1 & - & - & - & - & - \\
\hline A. nebulosus & - & - & - & 1 & - & 2 & - & 5 & 4 \\
\hline S. clarkii & - & - & - & - & - & 14 & - & 42 & - \\
\hline$U$. bicarinatus & - & - & - & 2 & & - & - & 15 & 18 \\
\hline P. callicephalus & - & - & - & - & 1 & - & 1 & - & - \\
\hline P. parviauriculatus & - & - & - & - & 1 & - & - & - & - \\
\hline A. costata & 30 & 163 & 22 & 2 & - & 7 & - & - & \\
\hline \multicolumn{10}{|l|}{$\mathrm{BP}$} \\
\hline E. kingii & 2 & - & 4 & - & 9 & - & - & - & - \\
\hline S. clarkii & - & - & - & 2 & - & 8 & - & 39 & 2 \\
\hline S. jarrovii & - & - & - & 132 & 41 & 13 & 7 & 16 & - \\
\hline S. lemosespinali & - & - & - & 7 & 1 & 12 & 3 & 23 & - \\
\hline S. virgatus & - & - & - & 14 & - & 1 & - & - & 1 \\
\hline P. callicephalus & - & - & - & - & 1 & - & 1 & - & - \\
\hline P. parviauriculatus & - & - & - & - & 1 & - & 1 & - & - \\
\hline
\end{tabular}


Santoyo-Brito \& Lemos-Espinal: Reparto de recursos en gremios de lagartijas

Cuadro 3. Valores de superposición de nicho espacial estimado mediante el índice de Pianka para las especies de saurofauna observadas en los microhábitats en el bosque tropical caducifolio, Cañón de Chínipas, Chihuahua.

\begin{tabular}{|c|c|c|c|c|c|c|c|c|c|c|}
\hline & $\mathbf{P t}$ & Hh & An & $\mathrm{Cm}$ & $\mathbf{H e}$ & Sa & Sc & Sn & Ub & Ac \\
\hline $\mathrm{Pt}$ & - & 0 & 0 & 0 & 0 & 0 & 0.02 & 0 & 0 & 0 \\
\hline $\mathrm{Hh}$ & & - & 0.15 & 0.19 & 0.64 & 0.20 & 0.20 & 0.19 & 0.21 & 0.88 \\
\hline An & & & - & 0.97 & 0.01 & 0.89 & 0.86 & 0.10 & 0.81 & 0.01 \\
\hline $\mathrm{Cm}$ & & & & - & 0.04 & 0.96 & 0.91 & 0.18 & 0.88 & 0.03 \\
\hline $\mathrm{He}$ & & & & & - & 0.04 & 0.03 & 0.22 & 0.02 & 0.89 \\
\hline $\mathrm{Sa}$ & & & & & & - & 0.92 & 0.04 & 0.93 & 0.06 \\
\hline $\mathrm{Sc}$ & & & & & & & - & 0.18 & 0.98 & 0.01 \\
\hline $\mathrm{Sn}$ & & & & & & & & - & 0.12 & 0.07 \\
\hline $\mathrm{Ub}$ & & & & & & & & & - & 0.02 \\
\hline Ac & & & & & & & & & & - \\
\hline
\end{tabular}

$\mathrm{Pt}=P$. tuberculosus, $\mathrm{Hh}=H$. horridum, $\mathrm{An}=A$. nebulosus, $\mathrm{Cm}=C$. macrolopha, $\mathrm{He}=H$. elegans, $\mathrm{Sa}=S$. albiventris, $\mathrm{Sc}=S$. clarkii, $\mathrm{Sn}=S$. nelsoni, $\mathrm{Ub}=U$. bicarinatus, $\mathrm{Ac}=A$. costata

Cuadro 4. Valores de amplitud de nicho espacial $(D s)$, estimado mediante la ecuación de Simpson, de la saurofauna observada en el bosque tropical caducifolio (BTC), bosque de encino (BE) y bosque de pino (BP), Cañón de Chínipas, Chihuahua.

\begin{tabular}{|c|c|c|c|}
\hline \multirow[b]{2}{*}{ Especie } & \multicolumn{3}{|c|}{ Asociación vegetal } \\
\hline & BTC & $\mathbf{B E}$ & $\mathbf{B P}$ \\
\hline A. costata & 0.22 & 0.09 & \\
\hline A. nebulosus & 0.19 & 0.14 & \\
\hline C. macrolopha & 0.28 & & \\
\hline H. elegans & 0.29 & & \\
\hline H. horridum & 0.18 & 0.07 & \\
\hline P. callicephalus & & 0.12 & 0.14 \\
\hline P. parviauriculatus & & 0 & 0.14 \\
\hline P. tuberculosus & 0 & & \\
\hline S. albiventris & 0.25 & & \\
\hline S. clarkii & 0.16 & 0.07 & 0.09 \\
\hline S. jarrovi & & & 0.17 \\
\hline S. kingii & & & 0.17 \\
\hline S. lemosespinali & & & 0.27 \\
\hline S. nelsoni & 0.02 & & \\
\hline S. virgatus & & & 0.04 \\
\hline U. bicarinatus & 0.13 & 0.15 & \\
\hline
\end{tabular}


Cuadro 5. Valores de superposición de nicho espacial estimado mediante el índice de Pianka para las especies de saurofauna observadas en los microhábitats en el bosque de encino ubicado en el Cañón de Chínipas, Chihuahua.

\begin{tabular}{|c|c|c|c|c|c|c|c|}
\hline & Hh & An & Sc & Ub & Pc & $\mathbf{P p}$ & Ac \\
\hline $\mathrm{Hh}$ & - & 0.04 & 0 & 0.02 & 0 & 0 & 0.92 \\
\hline An & & - & 0.79 & 0.93 & 0 & 0 & 0.01 \\
\hline $\mathrm{Sc}$ & & & - & 0.60 & 0 & 0 & 0.01 \\
\hline $\mathrm{Ub}$ & & & & - & 0 & 0 & 0.00 \\
\hline $\mathrm{Pc}$ & & & & & - & 0.70 & 0 \\
\hline $\mathrm{Pp}$ & & & & & & - & 0 \\
\hline Ac & & & & & & & - \\
\hline
\end{tabular}

$\mathrm{Hh}=H$. horridum, $\mathrm{An}=A$. nebulosus, $\mathrm{Sc}=S$. clarkii, $\mathrm{Ub}=$ U.bicarinatus, $\mathrm{Pc}=P$. callicephalus, $\mathrm{Pp}=P$. parviauriculatus, $\mathrm{Ac}=$ A. costata.

Cuadro 6. Valores de superposición de nicho espacial estimado mediante el índice de Pianka para las especies de saurofauna observada en los microhábitats en el bosque de pino ubicado en el Cañón de Chínipas, Chihuahua.

\begin{tabular}{|c|c|c|c|c|c|c|c|}
\hline & Ek & Sc & $\mathbf{S j}$ & SI & $\mathbf{S v}$ & Pc & $\mathbf{P p}$ \\
\hline Ek & - & 0 & 0.26 & 0.03 & 0 & 0.63 & 0.63 \\
\hline $\mathrm{Sc}$ & & - & 0.17 & 0.93 & 0.06 & 0 & 0 \\
\hline $\mathrm{Sj}$ & & & - & 0.39 & 0.94 & 0.24 & 0.24 \\
\hline $\mathrm{Sl}$ & & & & - & 0.28 & 0.10 & 0.10 \\
\hline $\mathrm{Sv}$ & & & & & - & 0 & 0 \\
\hline $\mathrm{Pc}$ & & & & & & - & 1 \\
\hline $\mathrm{Pp}$ & & & & & & & - \\
\hline
\end{tabular}

$\mathrm{Ek}=$ E. kingii, $\mathrm{Sc}=S$. clarkii, $\mathrm{Sj}=S$. jarrovi, $\mathrm{Sl}=S$. lemosespinali, $\mathrm{Sv}=S$. virgatus, $\mathrm{Pc}=P$. callicephalus, $\mathrm{Pp}=P$. parviauriculatus

Uso de tiempo en el BTC. De las diez especies registradas sólo tres, Ctenosaura macrolopha, P. tuberculosus y S. nelsoni, estuvieron presentes durante todos los horarios de muestreo. El horario en el que se observó mayor número de especies fue de 12:00 a 14:59 h con diez especies registradas. El horario en el cual se registró el menor número de especies fue de 08:00- 08:59 h con tres (Cuadro 7). Al evaluar el traslape se encuentran valores que van de 0 a 0.94 (Cuadro 8). Para las 
pseudocomunidades la media del traslape observado $=0.63$ y la media del traslape simulado $=0.47$. La probabilidad del traslape observado $\geq$ esperado $=0.000$. De manera general, el valor de la amplitud de nicho fue alto; en cinco de las diez especies se estimó un $D_{s}$ de por lo menos 0.5 (Cuadro 9).

Cuadro 7. Número de registros de las especies de saurofauna observadas por horarios de actividad en el bosque tropical caducifolio (BTC), bosque de encino (BE) y bosque de pino (BP), Cañón de Chínipas, Chihuahua.

\begin{tabular}{|c|c|c|c|c|c|c|c|c|c|c|c|}
\hline \multicolumn{12}{|c|}{ Horario } \\
\hline $\begin{array}{l}0 \\
\mathscr{n} \\
\dot{0} \\
1 \\
1 \\
0 \\
\dot{\infty} \\
0\end{array}$ & $\begin{array}{l}\stackrel{8}{0} \\
\ddot{8} \\
1 \\
8 \\
\dot{8}\end{array}$ & $\begin{array}{l}0 \\
0 \\
0 \\
0 \\
1 \\
o \\
0 \\
0\end{array}$ & 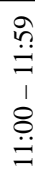 & 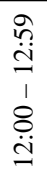 & 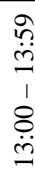 & $\begin{array}{l}\mathscr{\vartheta} \\
\stackrel{ \pm}{ \pm} \\
1 \\
8 \\
\dot{\Xi}\end{array}$ & 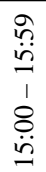 & 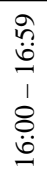 & 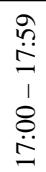 & $\begin{array}{l}\stackrel{0}{0} \\
\infty \\
1 \\
1 \\
o \\
\dot{0} \\
\dot{0}\end{array}$ & 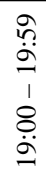 \\
\hline
\end{tabular}

BTC

A. costata

A. nebulosus

C. macrolopha

H. elegans

H. horridum

P. tuberculosus

S. albiventris

S. clarkii

S. nelsoni

$U$. bicarinatus

$\mathrm{BE}$

A. costata

A. nebulosus

H. horridum

P. callicephalus

P. parviauriculatus

S. clarkii

U. bicarinatus

BP

E. kingii

P. callicephalus

P. parviauriculatus

S. clarkii

S. jarrovii

S. lemosespinali

S. virgatus

$\begin{array}{cccccccccccc}- & 3 & 19 & 62 & 74 & 49 & 17 & 38 & 27 & 2 & - & - \\ - & - & 12 & 9 & 7 & 2 & 16 & - & - & - & - & - \\ 3 & 21 & 39 & 42 & 10 & 11 & 10 & 16 & 5 & 2 & 2 & 2 \\ - & 3 & 30 & 34 & 32 & 46 & 31 & 11 & 5 & 2 & - & - \\ - & - & - & - & 2 & 3 & 2 & - & - & - & 1 & 2 \\ 2 & 1 & 3 & 2 & 2 & 4 & 1 & 2 & 1 & 1 & 1 & 1 \\ - & 4 & 49 & 42 & 18 & 21 & 43 & 29 & 19 & 6 & 2 & 2 \\ - & 1 & 2 & 5 & 22 & 13 & 19 & 25 & 11 & 7 & 11 & 3 \\ 1 & 4 & 2 & 8 & 23 & 22 & 14 & 25 & 21 & 15 & 6 & 4 \\ - & - & 2 & 3 & 2 & 5 & 1 & 40 & 2 & 1 & 1 & 2\end{array}$


Cuadro 8. Valores de superposición de nicho temporal estimado mediante el índice de Pianka para las especies de sauriofauna en los microhábitats en el bosque tropical caducifolio, Cañón de Chínipas, Chihuahua.

\begin{tabular}{|c|c|c|c|c|c|c|c|c|c|c|}
\hline & $\mathbf{P t}$ & Hh & An & $\mathbf{C m}$ & He & $\mathbf{S a}$ & Sc & Sn & Ub & Ac \\
\hline $\mathrm{Pt}$ & - & 0.66 & 0.58 & 0.76 & 0.86 & 0.78 & 0.70 & 0.77 & 0.44 & 0.78 \\
\hline $\mathrm{Hh}$ & & - & 0.51 & 0.26 & 0.76 & 0.48 & 0.66 & 0.67 & 0.13 & 0.62 \\
\hline An & & & - & 0.72 & 0.80 & 0.87 & 0.53 & 0.44 & 0.09 & 0.60 \\
\hline $\mathrm{Cm}$ & & & & - & 0.76 & 0.87 & 0.46 & 0.49 & 0.35 & 0.70 \\
\hline $\mathrm{He}$ & & & & & - & 0.87 & 0.69 & 0.71 & 0.29 & 0.87 \\
\hline $\mathrm{Sa}$ & & & & & & - & 0.70 & 0.68 & 0.44 & 0.76 \\
\hline $\mathrm{Sc}$ & & & & & & & - & 0.94 & 0.66 & 0.78 \\
\hline $\mathrm{Sn}$ & & & & & & & & - & 0.60 & 0.82 \\
\hline $\mathrm{Ub}$ & & & & & & & & & - & 0.45 \\
\hline Ac & & & & & & & & & & - \\
\hline
\end{tabular}

$\mathrm{Pt}=P$. tuberculosus, $\mathrm{Hh}=H$. horridum, $\mathrm{An}=A$. nebulosus, $\mathrm{Cm}=C$. macrolopha, $\mathrm{He}=H$. elegans, $\mathrm{Sa}=S$. albiventris, $\mathrm{Sc}=S$. clarkii, $\mathrm{Sn}=S$. nelsoni, $\mathrm{Ub}=U$. bicarinatus, $\mathrm{Ac}=A$. costata

Cuadro 9. Valores de amplitud de nicho temporal ( $D s)$, estimado mediante la ecuación de Simpson, de la saurofauna observada en el bosque tropical caducifolio (BTC), bosque de encino (BE) y bosque de pino (BP) u, Cañón de Chínipas, Chihuahua.

\begin{tabular}{|c|c|c|c|}
\hline \multirow[b]{2}{*}{ Especies } & \multicolumn{3}{|c|}{ Asociación vegetal } \\
\hline & BTC & $\mathbf{B E}$ & $\mathbf{B P}$ \\
\hline A. costata & 0.43 & 0.58 & \\
\hline A. nebulosus & 0.56 & 0.41 & \\
\hline C. macrolopha & 0.46 & & \\
\hline H. elegans & 0.45 & & \\
\hline H. horridum & 0.32 & 0.16 & \\
\hline P. callicephalus & & 0.1 & 0.1 \\
\hline P. parviauriculatus & & 0 & 0.1 \\
\hline P. tuberculosus & 0.76 & & \\
\hline S. albiventris & 0.53 & & \\
\hline S. clarkii & 0.56 & 0.27 & 0.33 \\
\hline S. jarrovi & & & 0.68 \\
\hline S. kingii & & & 0.47 \\
\hline S. lemosespinali & & 0.20 & \\
\hline S. nelsonis & 0.63 & & \\
\hline S. virgatus & & & 0.35 \\
\hline U. bicarinatus & 0.10 & 0.24 & \\
\hline
\end{tabular}


Uso de tiempo en el BE. No se registró especie alguna ocupando todos los horarios de muestreo, Aspodoscelis costata se observó en nueve, Anolis nebulosus en siete, Heloderma horridum en tres, $P$. callicephalus en dos, $P$. parviauriculatus en uno, $S$. clarkii en siete y Urosaurus bicarinatus en cuatro. El horario ocupado por el mayor número de especies fue de 13:00 a 13:59 h y el de menor número fue 9:00 a 9:59 h y 19:00 a 19:59 h, ambos con una (Cuadro 7). Los valores estimados del traslape temporal van de 0 a 0.82 (Cuadro 10), el valor más alto se presentó entre $U$. bicarinatus y S. clarkii. Para las pseudocomunidades la media del traslape observado $=0.27$ y la media del traslape simulado $=0.28$. La probabilidad de que el traslape simulado $\geq$ esperado $=0.519$. De manera general, el valor de amplitud de nicho para las lagartijas registradas en el BE es bajo, indicando así la tendencia especialista (Cuadro 9).

Uso de tiempo en el BP. No se observó alguna especie en todos los horarios de muestreo. Sceloporus jarrovii se registró en once de los doce horarios. Los horarios ocupados por el mayor número de especies fueron 13:00 a 13:59 h y 15:00 a 15:59 h con seis y los de menor número 18:00 a 18:59 h y 19:00 a 19:59 h, ambos con una (Cuadro 7). Los resultados de traslape varían de 0 a 0.83 (Cuadro 11). Para las pseudocomunidades la media del traslape observado $=0.52$ y la media del traslape esperado $=0.36$. La probabilidad de que el traslape observado $\geq$ esperado $=0.001$. Respecto a la amplitud de nicho en el BP, organismos de cinco de las siete especies observadas presentaron valores menores a 0.40 , indicando así la tendencia especialista (Cuadro 9).

Cuadro 10. Valores de superposición de nicho temporal estimado mediante el índice de Pianka para las especies de saurofauna observadas en los microhábitats en el bosque de encino, Cañón de

Chínipas, Chihuahua.

\begin{tabular}{|c|c|c|c|c|c|c|c|}
\hline & Hh & An & Sc & Ub & Pc & Pp & Ac \\
\hline $\mathrm{Hh}$ & - & 0.07 & 0.01 & 0 & 0.28 & 0 & 0.02 \\
\hline An & & - & 0.36 & 0.27 & 0.40 & 0.37 & 0.79 \\
\hline $\mathrm{Sc}$ & & & - & 0.82 & 0.07 & 0.20 & 0.71 \\
\hline $\mathrm{Ub}$ & & & & - & 0 & 0.04 & 0.58 \\
\hline $\mathrm{Pc}$ & & & & & - & 0 & 0.42 \\
\hline $\mathrm{Pp}$ & & & & & & - & 0.27 \\
\hline $\mathrm{Ac}$ & & & & & & & - \\
\hline
\end{tabular}

$\mathrm{Hh}=H$. horridum, $\mathrm{An}=A$. nebulosus, $\mathrm{Sc}=S$. clarkii, $\mathrm{Ub}=$ U.bicarinatus, $\mathrm{Pc}=P$. callicephalus, $\mathrm{Pp}=P$. parviauriculatus, $\mathrm{Ac}=A$. costata . 
Cuadro 11. Valores de superposición de nicho temporal estimado mediante el índice de Pianka para las especies de saurofauna observadas en los microhábitats en el bosque de pino, Cañón de Chínipas, Chihuahua.

\begin{tabular}{|c|c|c|c|c|c|c|c|}
\hline & Ek & Sc & $\mathbf{S j}$ & SI & $\mathbf{S v}$ & Pc & $\mathbf{P p}$ \\
\hline $\mathrm{Ek}$ & - & 0.78 & 0.73 & 0.72 & 0.83 & 0.45 & 0.11 \\
\hline $\mathrm{Sc}$ & & - & 0.78 & 0.85 & 0.82 & 0.34 & 0.31 \\
\hline $\mathrm{Sj}$ & & & - & 0.63 & 0.76 & 0.37 & 0.52 \\
\hline S1 & & & & - & 0.82 & 0.16 & 0.42 \\
\hline $\mathrm{Sv}$ & & & & & - & 0.09 & 0.47 \\
\hline $\mathrm{Pc}$ & & & & & & - & 0 \\
\hline $\mathrm{Pp}$ & & & & & & & - \\
\hline
\end{tabular}

$\mathrm{Ek}=$ E. kingii, $\mathrm{Sc}=S$. clarkii, $\mathrm{Sj}=S$. jarrovi, $\mathrm{Sl}=S$. lemosespinali, $\mathrm{Sv}=S$. virgatus, $\mathrm{Pc}=P$. callicephalus, $\mathrm{Pp}=$ P. parviauriculatus

\section{DISCUSIÓN}

En el contexto del reparto de recursos la importancia de cada recurso difiere intra e interespecíficamente y en particular la mayoría de los reptiles simpátricos, con excepción de las serpientes, el hábitat o espacio es la primera dimensión que dividen (Toft 1985). Por ejemplo, especies simpátricas de lagartijas fraccionan dicho recurso en una gran variedad de hábitats (Schoener 1970; Pianka 1986) en relación con el grado de complejidad estructural de los mismos (Pianka 1975). La heterogeneidad en las tres asociaciones vegetales determinadas para el Cañón de Chínipas permitió reconocer el uso de nueve microhábitats, fácilmente identificables, en los que se registraron individuos de 16 especies de saurios, en total. Es de considerar que cuanto más heterogéneo sea el hábitat las especies de lagartijas simpátricas ocuparán nichos espaciales diferentes (Pianka 1973; Ortega et al. 1982), en consecuencia se esperaba que el traslape de nicho en el gremio estudiado fuera bajo. No obstante, los resultados del análisis de traslape indicaron que para algunos pares de especies los valores son altos; por ejemplo, $O_{j k}=0.98$ entre Sceloporus clarkii/Urosaurus bicarinatus, ubicado en BTC, $O_{j k}=0.93$ entre Heloderma horridum/Aspidoscelis costata ubicado en BE y $O_{j k}=0.94$ entre $S$. jarrovi/S. virgatus en BE. Sin embargo, los resultados generados en el mismo análisis para las pseudocomunidades de las tres asociaciones vegetales no sugieren la presencia de la posible competencia por el recurso. Por otra parte, los resultados del análisis de amplitud de nicho indican que los individuos de las especies estudiadas tienden a ser especialistas, siendo 0.29 el valor obtenido más alto.

El tiempo es el recurso menos importante cuando se toman en cuenta sólo lagartijas diurnas o nocturnas (Vitt \& Carvalho 1995), ya que la diferencia en los horarios de actividad conlleva el traslape nulo (Pianka 1973). Sin duda, la división 
del recurso tiempo se debe en parte a factores fisiológicos (Toft 1985) que repercuten en el uso del recurso; por ejemplo, los reptiles, al ser ectotermos, tienen acceso a un mayor intervalo de temperaturas que se refleja en el horario de actividad. En los primeros dos horarios de muestreo se registró por lo menos $25 \%$ del total de las especies encontradas en cada asociación vegetal $(\mathrm{BTC}=3 / 10 ; \mathrm{BE}=3 / 7 ; \mathrm{BP}=5 / 7$ ) y a partir de las 11:00 hasta las 17:59 h en cada asociación se registró a más de la mitad de las especies, incluso en el BTC se registraron todas las especies. Generalizando, a mediodía se observó el mayor número de especies, resultado que coincide con los de otros estudios realizados con diferentes especies de lagartijas (Vitt \& Carvalho 1995; García-de la Peña et al. 2007). Con base en el número de especies activas durante un mismo horario se sugiere que, de los dos recursos evaluados, el temporal es el de menor importancia (Vitt \& Carvalho 1995). Sin embargo, los resultados obtenidos del análisis de traslape de nicho temporal para las pseudocomunidades, indican que la competencia por este recurso puede estar operando para el gremio de lagartijas del BTC y del BP, pero no en el gremio del BE. Por otra parte, los valores estimados para la amplitud de nicho temporal son bajos, lo que muestra que los individuos de las especies de lagartijas presentes en el Cañón de Chínipas tienden a ser especialistas, lo que implica considerar al recurso tiempo como el más importante.

Con base en lo antes expresado se presenta la condición que sustenta la teoría del nicho, es decir, si la amplitud de nicho en alguna dimensión es baja en otra tenderá a ser alta (Pianka 1986), explicando así que organismos que pueden hacer uso más amplio de cierto recurso fraccionan en mayor grado otro recurso (Toft 1985). Con la información obtenida es posible advertir por qué coexisten las especies que conforman los gremios en las asociaciones vegetales del Cañón de Chínipas, ya que las formas en que las especies utilizan los recursos disponibles son factor determinante de la diversidad de especies (Pianka 1974) así como de su coexistencia y reflejan necesidades ecológicas específicas de las mismas. Sin embargo, un análisis más profundo debe tomar en cuenta la relación existente entre los recursos; no obstante, en específico para las lagartijas las relaciones entre los recursos se consideran más o menos independientes aunque algunas veces muestran interacción (Pianka 1973).

Los resultados del presente estudio son la primera aproximación al conocimiento de algunos aspectos primordiales de la ecología de los gremios de lagartijas que habitan el Cañón de Chínipas.

AGRADECIMIENTOS. Al Consejo Nacional de Ciencia y Tecnología y al Colegio de Postgraduados; sin dudar, dos grandes instituciones. A Dulce Ávila-Nájera y Cynthia Vargas. A los árbitros anónimos. Apoyo para el desarrollo del trabajo de campo fue proporcionado a Julio Lemos-Espinal por el Consejo Nacional de Ciencia y Tecnología (CONACyT) a través del proyecto - Apoyos Complementarios 52557, y la Comisión Nacional para el Conocimiento y Uso de la Biodiversidad (CONABIO) a través del proyecto EE009. 


\section{LITERATURA CITADA}

Altamirano, A.T. \& C.R. García. 1989. Análisis del nicho trófico y papel ecológico de la comunidad herpetológica de Alvarado, Veracruz, México. Umbrales, 1:20-26.

Altamirano, A.T., R.M. Vidal, C.R. García \& D.M. Ferríz. 1990. Análisis del nicho trófico y espacial de algunas especies de anfibios y reptiles de Alvarado, Veracruz. Revista de Zoología, 2:3-13.

Altamirano, A.T., C.R. Vizcaya, C.R. García \& S.M. Soriano. 1992. Uso de espacio y ciclo de actividad entre especies de lagartijas simpátricas. Revista de Zoología, 3:5-18.

Álvarez, A.T. \& M.S. Sarabia. 2006. Espectro alimentario de Aspidoscelis deppii (Sauria: Teiidae). Revista de Zoología, 17:39-45.

Álvarez, A.T. \& M. Sarabia, S. 2007. Espectro alimentario de Aspidoscelis guttata (Reptilia: Teiidae). Revista de Zoología, 18:19-26.

Arriaga, L., J.M. Espinoza, C. Aguilar, E. Martínez, L. Gómez \& E. Loa (coordinadores). 2000. Regiones Terrestres Prioritarias de México. Comisión Nacional para el Conocimiento y Uso de la Biodiversidad. México. Consultado en línea en 2009. http://www.conabio.gob.mx/conocimiento/ regionalizacion/doctos/terrestres.html

García-de la Peña, C., H. Gadsden, A.J. Contreras Balderas \& G. Castañeda. 2007. Ciclos de actividad diaria y estacional de un gremio de saurios en las dunas de arena de Viesca, Coahuila, México. Revista Mexicana de Biodiversidad, 78:141-147.

Gotelli, N.J. \& G.L. Entsminger. 2001. EcoSim: Null models software for ecology. Version 6.0 Acquired Intelligence Inc. \& Kesey-Bear. http:homepage.together.net/ gentsmin/ecosim.htm.

Hurlbert, S.H. 1978. The measurement of niche overlap and some relatives. Ecology, 59:67-77.

Joern, A. \& L.R. Lawlor. 1980. Food and microhabitat utilization by grasshoppers from arid grasslands: comparison with neutral models. Ecology, 63:591-599.

Lara, L.R. \& R. Castro-Franco. 1991. Introducción a la ecología de Cnemidophorus sacki gigas. Resúmenes del segundo encuentro regional de investigadores en Flora y Fauna Zona V de Anuies. Pachuca, Hgo.: 70.

Lawlor, L.R. 1980. Overlap, similarity, and competition coefficients. Ecology, 61:245-251.

Lemos-Espinal, J.A. \& J. Franco-López. 1984. Repartición del recurso espacio en una comunidad de anfibios y reptiles de la vertiente oriental del volcán Iztaccihuatl, Puebla. Ciencia Forestal, 50:4456.

Lemos-Espinal, J.A. \& H.M. Smith. 2007. Anfibios y reptiles del estado de Chihuahua, México/Amphibians and reptiles of the state of Chihuahua, México. CONABIO, México.

Luiselli, L. 2008. Resource partitioning in freshwater turtle communities: A null model-meta-analysis of available data. Acta Oecologica, 34:80-88.

Macip-Ríos, R. \& A. Muñoz-Alonso. 2008. Diversidad de lagartijas en cafetales y bosque primario en el Soconusco chiapaneco. Revista Mexicana de Biodiversidad, 79:185-195

Oliveira M.D., G. Corrêa-Acosta \& G.R. Colli. 2006. Ecology o fan amazonian savanna lizard assemblage in Monte Alegre, Prá state, Brazil. South American Journal of Herpetology, 1:61-71.

Ortega A., M. E. Maury \& R. Barbault. 1982. Spatial organization and habitat partitioning in a mountain lizard community of Mexico. Acta Oecologica/Oecologia Generalis, 3:323-330.

Ortega, A., A. González-Romero \& R. Barbault. 1992. The ecological organization or highly diverse desert lizard community in Mexico. Pp. 207-239. In: V. Sokolov, G. Halffter and A. Ortega (Eds). Vertebrate ecology in arid zones of Mexico and Asia. Instituto de Ecología A.C. - CIBNOR- MABUNESCO.

Ortega, A., A. González-Romero \& R. Barbault 1994. Structure and Functioning of a desert lizard community at El Pinacate, Sonora, México, Part II. The Herptile, 20:68-79. 
Ortega, A., A. González-Romero \& R. Barbault 1995. Food analysis and resource partitioning in a lizard guild of the Sonora Desert, México. Journal of Arid Environments, 29:367-382.

Pianka, E.R. 1969. Sympatry of desert lizards (Ctenotus) in Western Australia. Ecology, 50:1012-1030.

Pianka, E.R. 1973. The structure of lizard communities. Annual Review of Ecology and Systematics, 4:53-74.

Pianka, E.R. 1974. Niche Overlap and Difusse Competition. Proceedings of the National Academy of Science, 7:2141-2145.

Pianka, E.R. 1975. Niche relations of desert lizards. Pp. 292-314. In: M.L. Cody and J.M. Diamond (Eds). Ecology and evolution of communities. Harvard University Press.

Pianka, E.R. 1986. Ecological phenomena in evolutionary perspective. Pp. 325-336. In: N. Polunin (Ed). Ecosystem Theory and Application. Wiley and Sons.

Pianka, E.R. 1994. Biodiversity of australian desert lizards. Pp. 258-281. In: C. Peng \& C.H. (Eds). Biodiversity and terrestrial ecosystems. Monographs series 14, Institute of Botany. Academica Sinica, Taipei.

Sale, P.F. 1974. Overlap in resource use, and interespecific competition. Oecologia, 17:245-256.

Schoener, T.W. 1968. The Anolis lizards of Bimini: resource partitioning in a complex fauna. Ecology, 49:704-726.

Schoener, T.W. 1970. Size patterns in West Indian Anolis lizards. II. Correlations with the size of particular sympatric species. Displacement and convergence. American Naturalist, 104: 155-174.

Schoener, T.W. 1974. Resource partitioning in ecological communities. Science, 185:27-39.

Schoener, T.W. 1977. Competition and the niche. Pp. 35-136. In: C. Gans and D.W. Tinkle (Eds). Biology of the Reptilia. Ecology and Behavior. Academic Press, London.

Schoener, T.W. 1986. Resource partitioning. Pp. 90-126. In: J Kikkawa. and D.J. Anderson (Eds). Community ecology, pattern and process. Blackwell Scientific Publications, Oxford.

Simpson, E.H. 1949. Measurement of diversity. Nature, 163: 688.

Toft, C.A. 1985. Resource partitioning in amphibians and reptiles. Copeia, 1985:1-21.

Vitt, L.J. \& C.M. Carvalho. 1995. Niche partitioning in a tropical wet season: Lizards in the Labrado area of northern Brazil. Copeia, 1995: 305-329.

Vitt, L.J. \& P.A. Zani. 1996. Organization of a taxonomically diverse lizard assemblage in Amazonian Ecuador. Canadian Journal of Zoology, 74:1313-1335.

Winemiller, K.O. \& E.R. Pianka. 1990. Organization in natural assemblages of desert lizards and tropical fishes. Ecological Monographs, 60:27-55. 